\title{
VIDEO LARYNGOSCOPY FOR ENDOTRACHEAL INTUBATION OF ADULT PATIENTS WITH SUSPECTED/ CONFIRMED COVID-19. A SYSTEMATIC REVIEW AND META-ANALYSIS OF RANDOMIZED CONTROLLED TRIALS
}

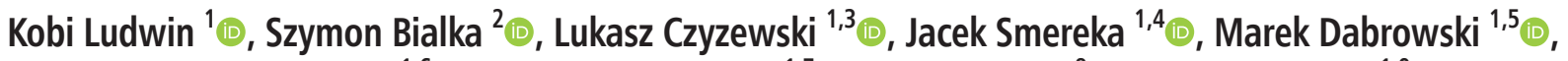

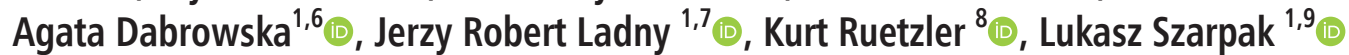 \\ ${ }^{1}$ Polish Society of Disaster Medicine, Warsaw, Poland \\ ${ }^{2}$ Department of Anaesthesiology and Critical Care, School of Medicine with Division of Dentistry in Zabrze, Medical University of Silesia, Zabrze, Poland \\ ${ }^{3}$ Department of Nephrology Nursing, Medical University of Warsaw, Poland \\ ${ }^{4}$ Department of Emergency Medical Service, Wroclaw Medical University, Wroclaw, Poland \\ ${ }^{5}$ Chair and Department of Medical Education, Poznan University of Medical Sciences, Poznan, Poland \\ ${ }^{6}$ Department of Medical Rescue, Chair of Emergency Medicine, Poznan University of Medical Sciences, Poznan, Poland \\ ${ }^{7}$ Clinic of Emergency Medicine, Medical University of Bialystok, Poland \\ ${ }^{8}$ Departments of Outcomes Research and General Anesthesia, Anesthesiology Institute, Cleveland Clinic, Cleveland, Ohio, USA \\ ${ }^{9}$ Comprehensive Cancer Center in Bialystok, Poland
}

\begin{abstract}
INTRODUCTION: During a pandemic, medical personnel while in contact with patients with suspected/confirmed COVID-19 should wear full personal protective equipment (PPE) for aerosol-generating procedures to reduce the risk of infection. Most studies of intubation in level C PPE conditions have been relatively small. Our aim is to quantify the available data on success rates in order to provide an evidence-based benchmark to gauge performance in the published literature.

MATERIAL AND METHODS: A structured literature search was performed with PubMed, Scopus, Embase, Web of Science, and Cochrane databases. The electronic database search was supplemented by searching Google Scholar and by back-searching the reference lists of identified studies for suitable articles. Data were evaluated and extracted by two independent reviewers on the basis of qualitative and quantitative variables of interest. Q statistic and $\mathrm{I}^{2}$ statistics were used to assess the heterogeneity between the studies.
\end{abstract}

RESULTS: Fifteen randomized controlled trials were included. The use of PPE during intubation as compared with intubation without PPE reduced intubation efficacy (90.0\% vs. $97.9 \% ; \mathrm{RR}=0.94 ; 95 \% \mathrm{Cl}: 0.90-0.99$; $\mathrm{p}<0.001)$ and increased the procedure time (MD $=7.73 ; 95 \% \mathrm{Cl}: 4.98-10.47 ; \mathrm{p}<0.001)$. Direct laryngoscopy compared with video laryngoscopes offered similar intubation success rate $(93.6 \%$ vs. $92.3 \% ; R R=0.99$; $95 \% \mathrm{Cl}: 0.97-1.02 ; \mathrm{p}=0.66)$ and shorter intubation time (MD $=63 ; 95 \% \mathrm{Cl}:-0.77-12.03 ; p=0.08)$. However, subgroup analysis showed that intubation with Macintosh blade video laryngoscopes was more effective than that with direct laryngoscopes ( $98.1 \%$ vs. $96.4 \% ; \mathrm{RR}=1.00 ; 95 \% \mathrm{Cl}: 0.97-1.03 ; \mathrm{p}=0.90$ ).

CONCLUSIONS: Our meta-analysis suggests that PPE reduces the effectiveness of endotracheal intubation. The use of direct laryngoscopy for intubating patients with suspected/confirmed COVID-19 by an intubator wearing level C PPE is associated with overall intubation time reduction and an increase in intubation success rate compared with video laryngoscopes. However, the findings suggest that Macintosh blade video 
laryngoscopes during endotracheal intubation with PPE may be an alternative to direct laryngoscopes. Video laryngoscopy can be helpful for less experienced personnel.

KEY WORDS: endotracheal intubation, laryngoscope, infected patient, COVID-19, personal protective equipment, meta-analysis

Disaster Emerg Med J 2020; 5(2): 85-97

\section{INTRODUCTION}

Endotracheal intubation is the gold standard for airway management in many clinical situations [1, 2]. Direct laryngoscopy with a Macintosh laryngoscope is still commonly performed in endotracheal intubation. Unsuccessful or prolonged endotracheal intubation can be associated with many serious complications, such as desaturation, sympathetic stimulation leading to hypertension and tachycardia and even hypoxemic cardiac arrest causing permanent neurological sequel or even death [3]. In light of this, intubation with video laryngoscopes has become more commonly performed. It has been reported that video laryngoscopes can provide improved laryngeal visualization as well as increased intubation success rate, especially in difficult airway patients $[4,5]$.

In the current SARS-CoV-2 pandemic, each patient under emergency medical conditions should be considered potentially infected. Therefore, medical personnel should wear specialist personal protective equipment (PPE), including full PPE for aerosol-generating procedures, respiratory protection preferably with an FFP3 filter, goggles, face shield, and gloves [6]. The need for this protection of medical personnel at high risk of contact with suspected/confirmed COVID-19 patients results from the fact that the new coronavirus SARS-CoV-2 spreads via droplets, contact, and natural aerosols from human to human [7]. Moreover, the coronavirus is highly infectious, as verified by recent epidemiological data. As of April 10, 2020, the reported number of confirmed infection cases equaled $1,777,612$. COVID-19 mortality is $6.1 \%$ and turns out lower than that in SARS or MERS, but the disease dynamics is very high. Patients with COVID-19, in severe cases, can progress rapidly and develop acute respiratory distress syndrome, septic shock, metabolic acidosis, and coagulopathy $[8,9]$. In any case of patient deterioration and acute respiratory distress syndrome development, intubation should be performed and mechanical ventilation implemented [10]. Endotracheal intubation and advanced resuscitation should also be applied in the case of sudden cardiac arrest in such a patient [11]. It is therefore clear that the use of full PPE may reduce the risk of virus transmission [9]. However, research shows that the effectiveness of medical procedures performed with a PPE suit may be reduced [12]. This also refers to endotracheal intubation. It is thus reasonable to evaluate the available studies concerning various methods of endotracheal intubation in order to search for the most effective method of airway management in patients with suspected/confirmed COVID-19.

Recently, several studies have evaluated the effect of video laryngoscopy compared with direct laryngoscopy performed in infectious patients by operators wearing level C PPE. With the aid of the increased power of meta-analytic methods, the goal of the present study was to review the relevant and available published randomized controlled trials (RCTs) to test the hypothesis that compared with direct laryngoscopy, the use of video laryngoscopy in infectious patients would increase the intubation success rate.

\section{MATERIAL AND METHODS}

The manuscript followed the recommendations of the Preferred Reporting Items for Systematic Reviews and Meta-Analyses (PRISMA) statement [13]. Before commencing the study, we agreed on the analysis methods and the inclusion and exclusion criteria to be applied. The protocol of this meta-analysis study has not been registered.

\section{Inclusion and exclusion criteria}

Studies were included if they met the following criteria: (1) RCT; (2) clinical, cadaver, or simulation trial; (3) intubation of an adult patient or a simulator; (4) comparison of intubation with different laryngoscopes with/without level C PPE; (5) reporting any of the following outcomes: intubation success rate, time to intubation, glottis visualization. Articles available only in abstract form and meeting reports were excluded. Studies in English were included.

\section{Search strategy}

A comprehensive literature search was performed with PubMed, Scopus, Embase, Web of Science, and 
Cochrane databases, from the inception of each database up to March 30, 2020. The following terms were used: "Macintosh laryngoscope" or "Miller laryngoscopes" or "laryngoscope" or "video laryngoscopy" or "endotracheal intubation" or "tracheal intubation" or "airway management" and "PPE" or "personal protective equipment" or "HazMat" or "Level C protective" or "CBRN" or "Chemical" or "toxic" or "infectious patient". The electronic database search was supplemented by searching Google Scholar and by back-searching the reference lists of identified studies for suitable articles.

\section{Data extraction}

Two authors (K.L. and J.S.) independently assessed each article to determine whether or not it met the criteria for inclusion. Disagreements between the authors regarding values or analysis assignments were resolved through discussion with a third researcher (L.S.), and the decision was taken by the majority of the researchers. The agreement with respect to study inclusion was assessed by using the Cohen kappa statistics [14]. We were careful to avoid the inclusion of data from duplicate publications. In any case of suspected data discrepancies, we contacted the relevant author directly. Each author also performed independent data abstraction using standardized data collection forms. Data extracted from eligible studies included the following characteristics: study and year, country, type of participants, a number of participants, type of devices applied for intubation, intubation with/without PPE, intubation time, and success of intubation. If outcomes were reported for more than one follow-up period, we used data for the longest follow-up in each trial.

\section{Quality assessment}

The quality of eligible trials was assessed by using the "risk of bias" tool in accordance with the Review Manager software, version 5.3 (RevMan; Cochrane Collaboration, Oxford, UK). Two authors (L.S. and K.J.F.) estimated the risk of bias in the following methodological domains: random sequence generation, allocation concealment, blinding of participants and personnel, blinding of outcome assessment, incomplete outcome data, selective reporting, others bias [15]. Each was graded "yes", "no", or "unclear", which reflected a high risk of bias, low risk of bias, and uncertain bias, respectively (Suppl. digital content 1). The review authors' judgments about each risk of bias item are provided in Suppl. digital content 2 .

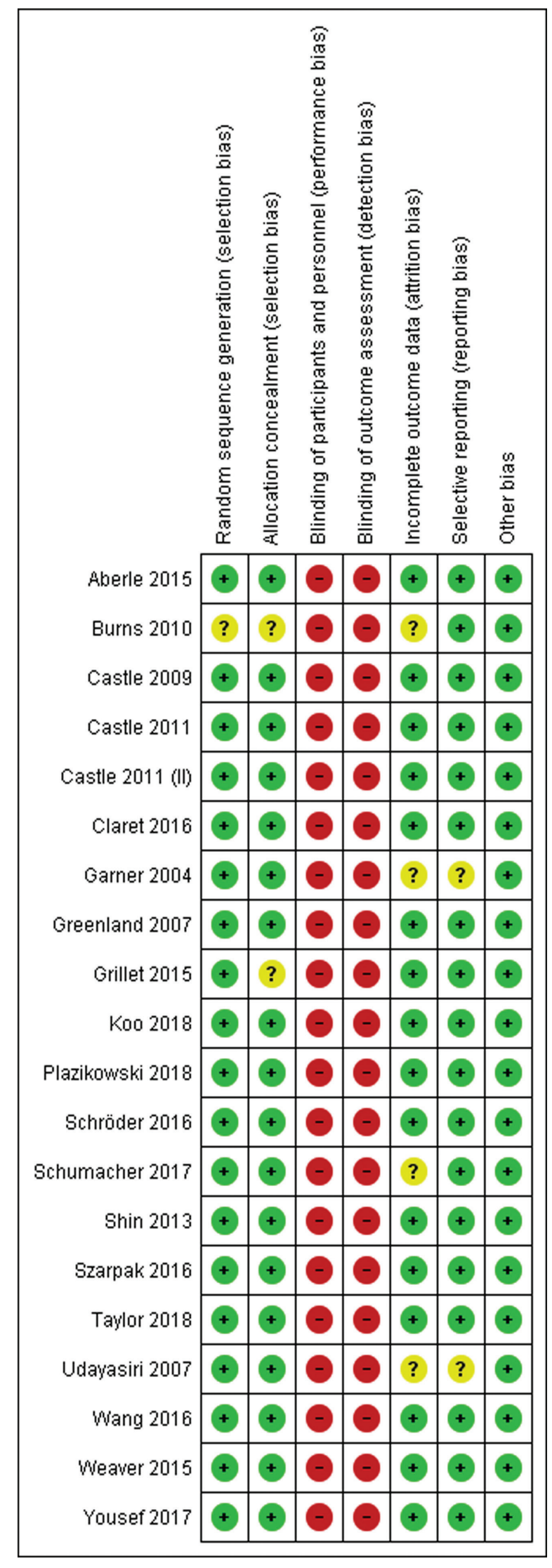

SUPPLEMENT 1. The risk of bias (Suppl. digital content 1) 


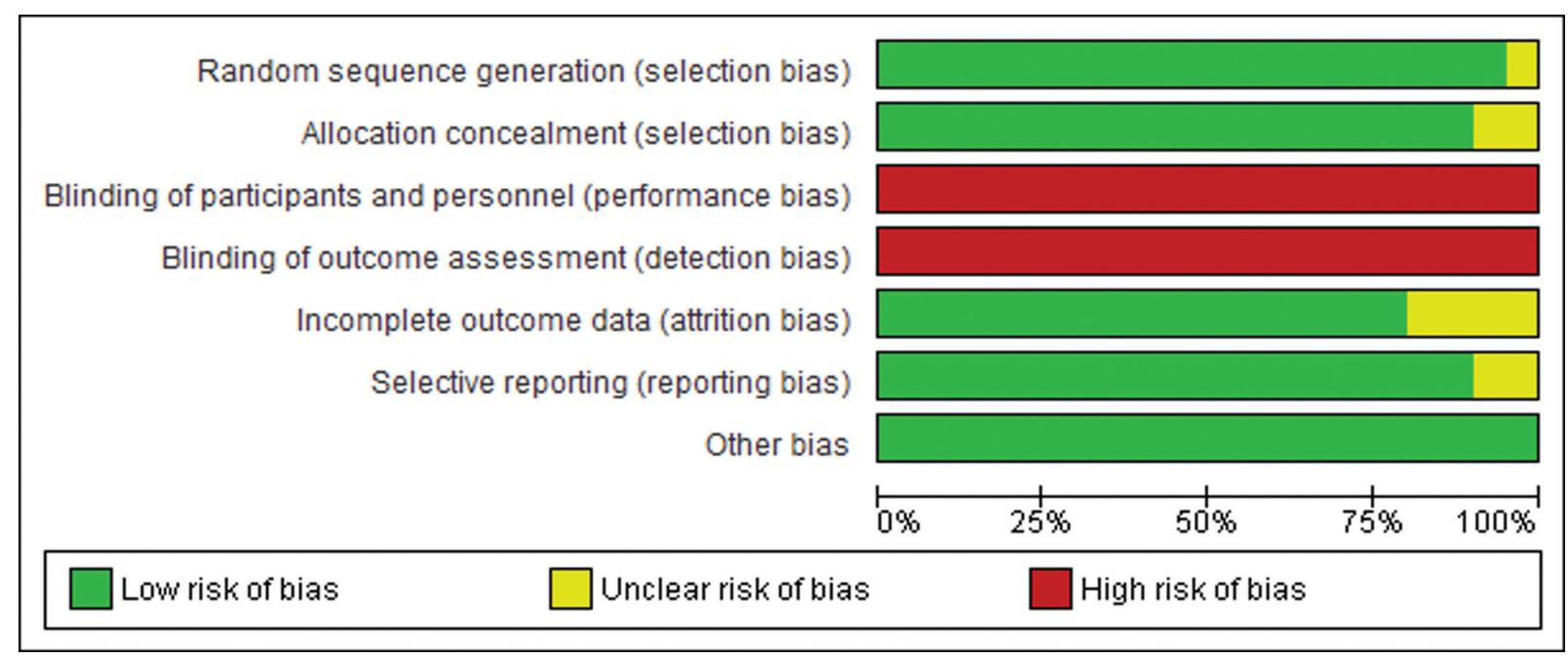

SUPPLEMENT 2. The review author's judgments about each risk of bias (Suppl. digital content 2)

\section{Data analysis}

For statistical analyses, we used the Review Manager (RevMan) software, version 5.3. Because there may be differences in the treatment effect between trials, especially those using different devices, we assumed a random-effects model. We employed the inverse-variance method for the continuous outcomes and the Mantel-Haenszel models for all dichotomous outcomes. We calculated mean differences (MD) for continuous measurements (time to intubation) and risk ratios (RR) for dichotomous outcomes (intubation success rate). All statistical variables were determined with 95\% confidence interval $(\mathrm{Cl})$ to estimate the range of plausible treatment effects. When the continuous outcome was reported in a study as median, range, and interquartile range, we estimated means and standard deviations using the formula described by Hozo et al. [16]. We quantified heterogeneity in each analysis by the tau-squared and I-squared statistics. Studies were subgrouped by the type of intubation devices. Heterogeneity was detected with the chi-squared test with n-1 degree of freedom, which was expressed as $\mathbf{l}^{2}$. Values of $I^{2}>50 \%$ and $>75 \%$ were considered to indicate moderate and significant heterogeneity among studies, respectively [16]. All p-values were tailed and considered statistically significant if $\mathrm{p}<0.05$.

\section{RESULTS \\ 1. Trial identification and characteristics}

Initially, 297 articles were identified for review based on our search of the electronic databases. Of these, 133 were excluded because they were not relevant.

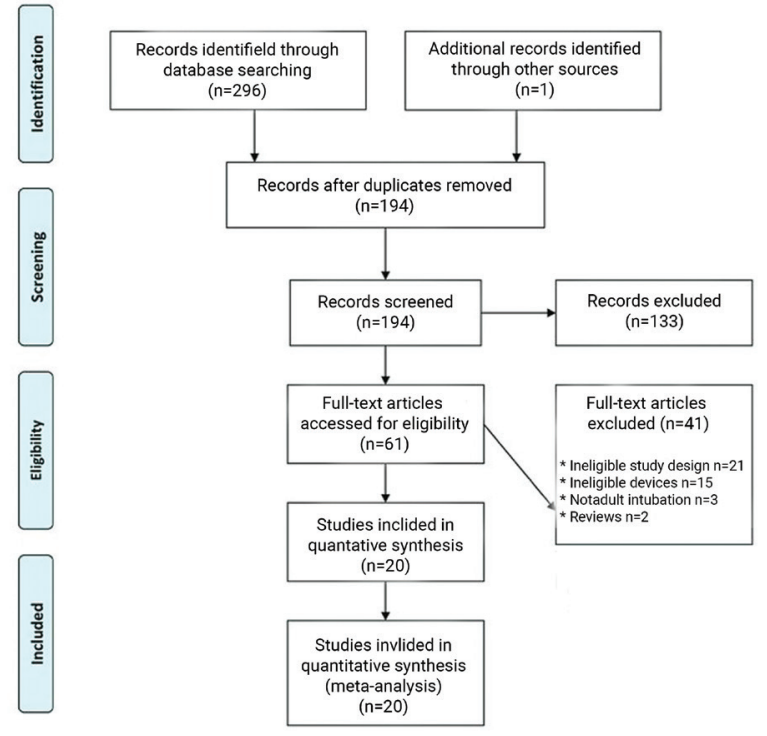

FIGURE 1. 20 studies with the inclusion criteria for data

The remaining 61 articles were carefully examined for meeting the inclusion criteria. Of those, 41 studies were excluded because they were not RCTs comparing direct laryngoscopy with video laryngoscopy $(n=21)$, provided comparisons between unrelated airway management devices $(n=15)$, did not refer to adult intubation $(n=3)$, were review articles $(n=2)$. Ultimately, 20 studies that met the inclusion criteria and contained the necessary data for the planned comparison were identified (Fig. 1). The details of the selected trials are summarized in Table 1. Among the 20 mentioned studies, two were cadaver studies $[17,18]$ and the others were simulation trials [12, 19-35]. 


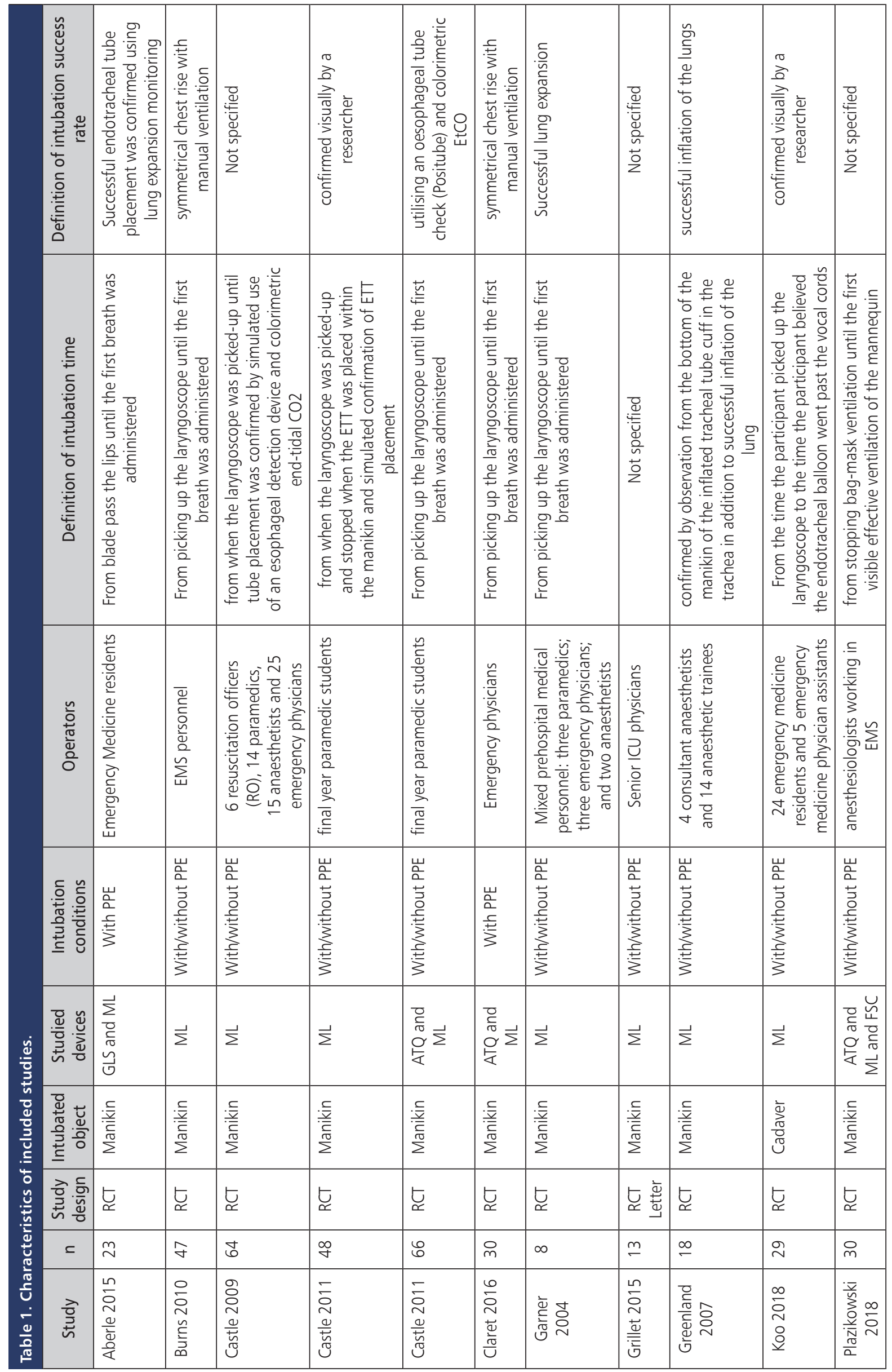




\begin{tabular}{|c|c|c|c|c|c|c|c|c|c|}
\hline 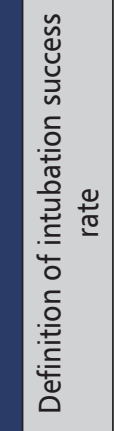 & 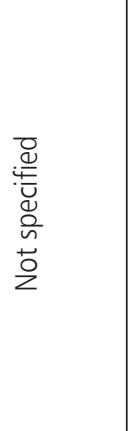 & 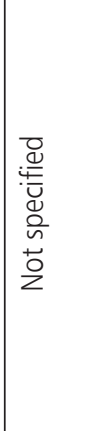 & 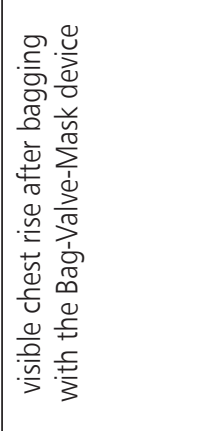 & 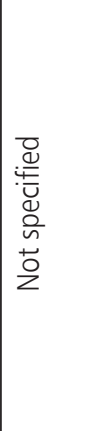 & 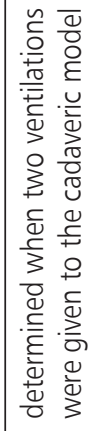 & 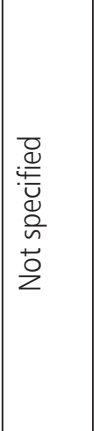 & 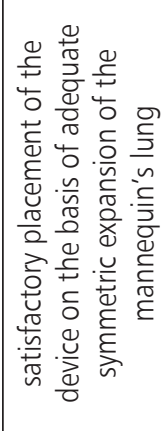 & 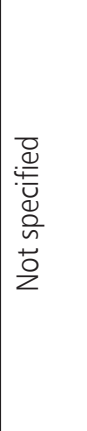 & 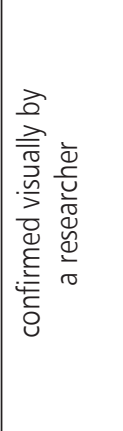 \\
\hline 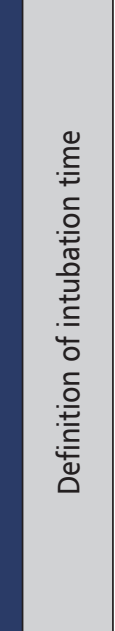 & 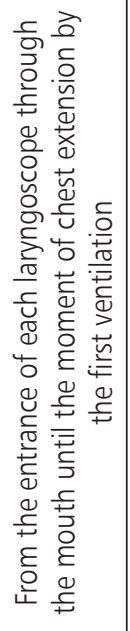 & 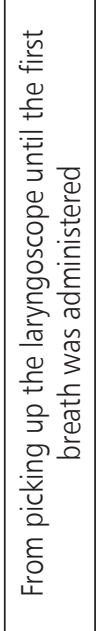 & 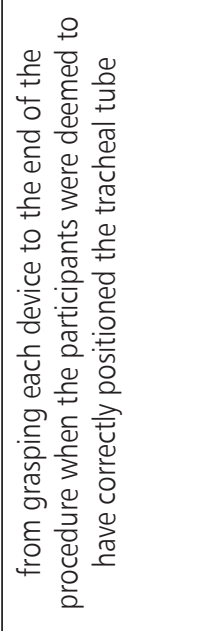 & 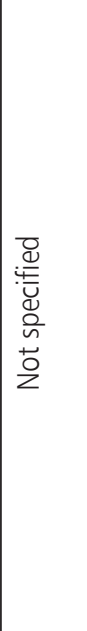 & 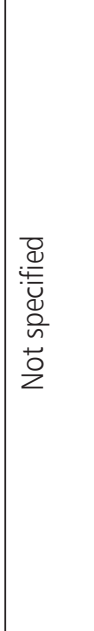 & 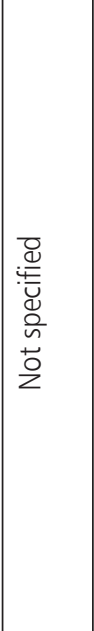 & 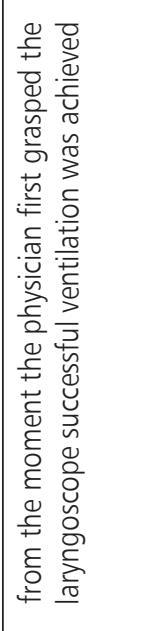 & 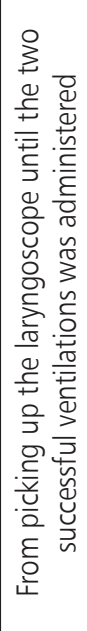 & 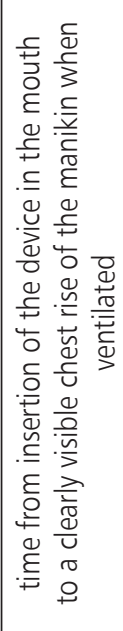 \\
\hline $\begin{array}{l}\frac{n}{0} \\
\frac{0}{0} \\
\frac{\pi}{0} \\
0\end{array}$ & 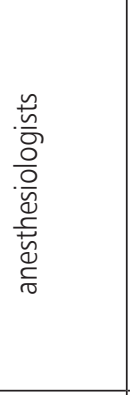 & 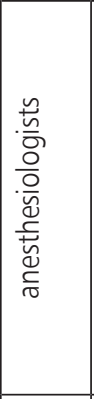 & 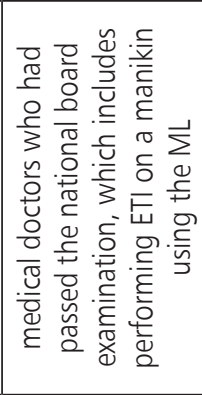 & 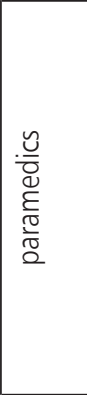 & 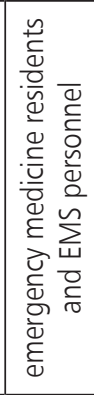 & 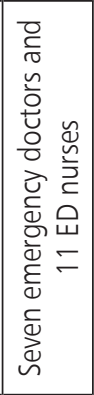 & 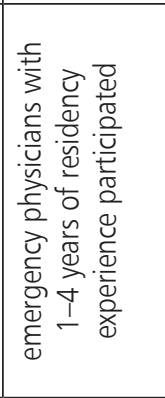 & 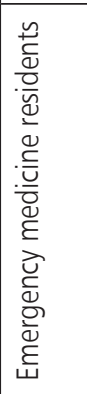 & 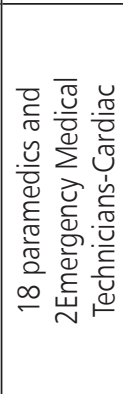 \\
\hline 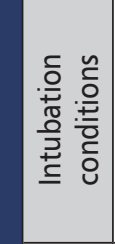 & $\begin{array}{l}\frac{w}{a} \\
\frac{a}{\alpha} \\
+\frac{5}{3} \\
3\end{array}$ & 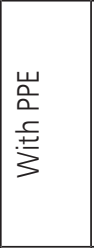 & 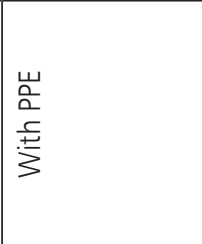 & 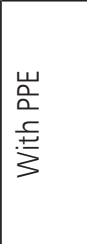 & 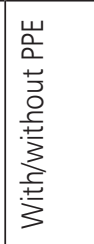 & & 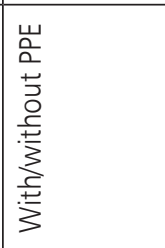 & 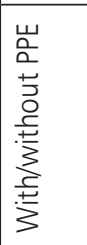 & 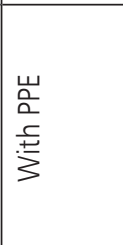 \\
\hline 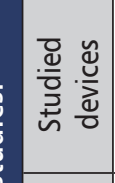 & 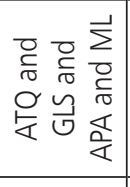 & $\vec{\Sigma}$ & $\sum_{\substack{\frac{D}{\pi} \\
\frac{\pi}{\pi}}}$ & 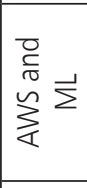 & 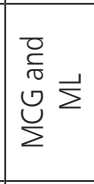 & & $\vec{\Sigma}$ & $\begin{array}{l}\vec{\sum} \\
0 \\
\frac{0}{0} \\
\tilde{v} \\
\tilde{v}\end{array}$ & 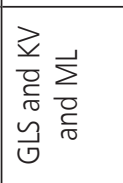 \\
\hline 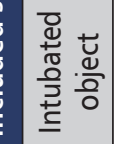 & 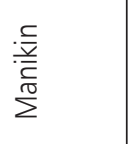 & 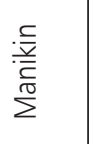 & 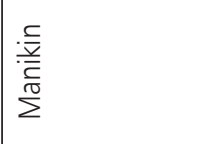 & 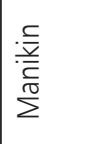 & \begin{tabular}{|l}
$\bar{y}$ \\
$\overline{0}$ \\
$\frac{\pi}{8}$ \\
$\overline{0}$
\end{tabular} & 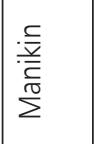 & 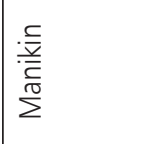 & 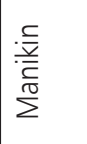 & 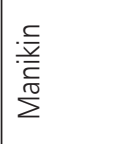 \\
\hline 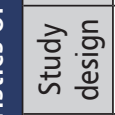 & $t_{\propto}$ & $\underset{ષ}{\varpi}$ & $\underset{\propto}{\longleftarrow}$ & 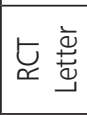 & 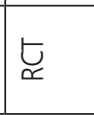 & $\underset{\propto}{ঢ}$ & $\underset{\propto}{E}$ & $\underset{\propto}{\longleftarrow}$ & 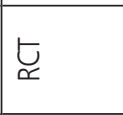 \\
\hline 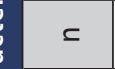 & $\mathscr{F}$ & $\stackrel{\circ}{m}$ & $\bar{m}$ & $\stackrel{\Re}{\forall}$ & $\stackrel{n}{\sim}$ & & 아 & $\hat{m}$ & $\stackrel{\sim}{\sim}$ \\
\hline $\begin{array}{l}\text { 흘 } \\
\text { 至 }\end{array}$ & 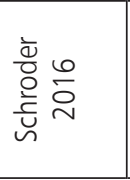 & 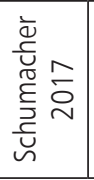 & 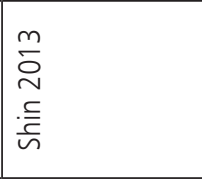 & 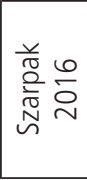 & 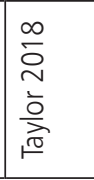 & 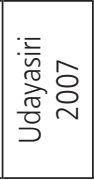 & \begin{tabular}{|l}
0 \\
$\frac{0}{2}$ \\
$N$ \\
0 \\
$\frac{1}{01}$ \\
3
\end{tabular} & 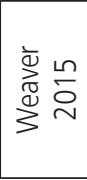 & 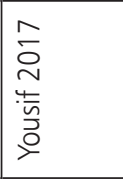 \\
\hline
\end{tabular}




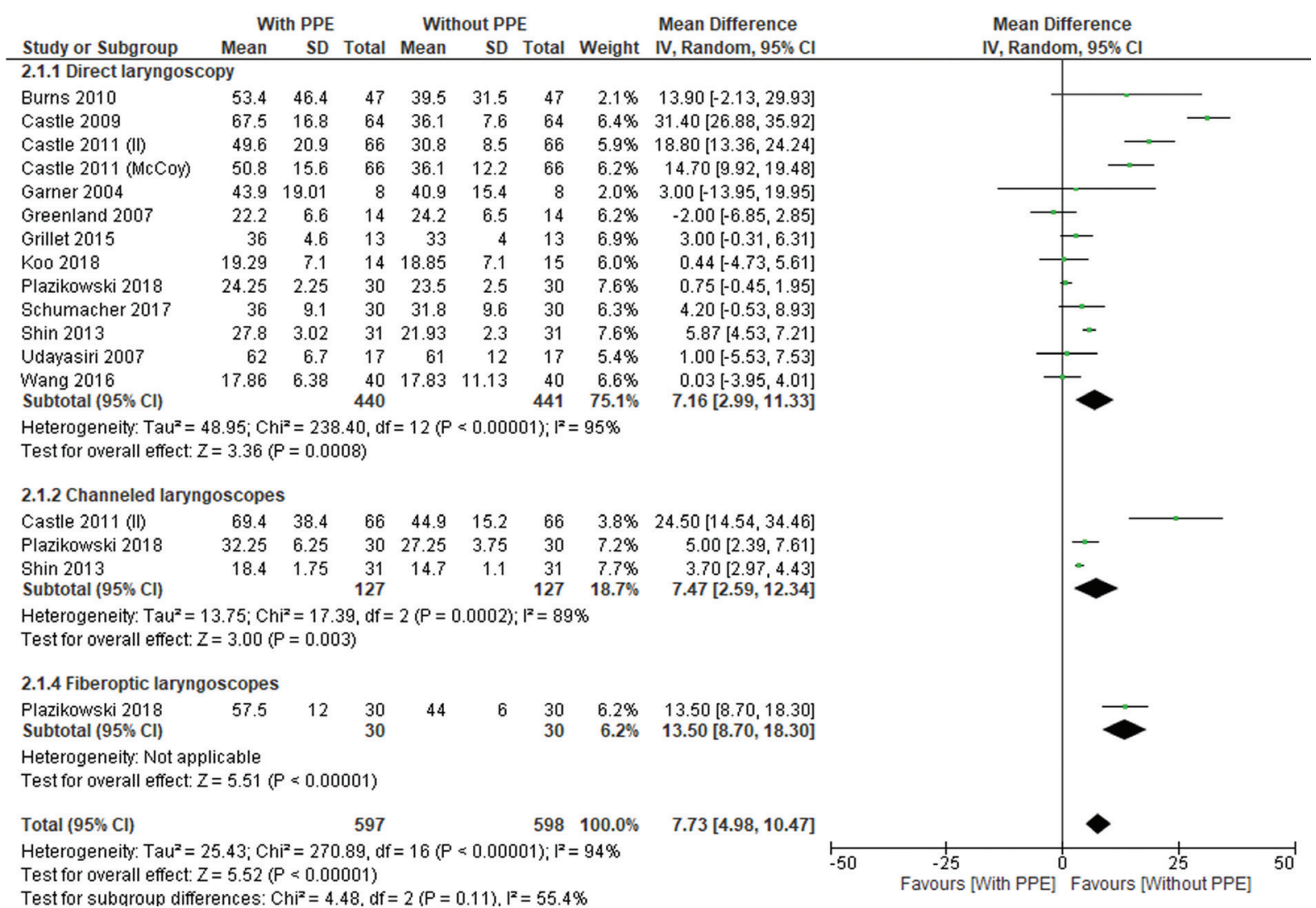

FIGURE 2. Time to intubation without PPE compared with PPE conditions

\section{Personal protective equipment impact on endotracheal intubation}

Thirteen studies with 1109 intubations reported impact of level C PPE on the duration of endotracheal intubation [12, 17, 20-22, 24-27, 29, 30, 32, 33]. Overall, time to intubation was shorter without PPE compared with PPE conditions (MD $=7,33 ; 95 \% \mathrm{Cl}$ : 4.98-10.47; $p<0.001$ ) (Fig. 2).

Subgroup analysis showed that the use of PPE extended all intubation techniques, including those applying direct laryngoscopes (MD $=7.16 ; 95 \% \mathrm{Cl}$ : 2.99-11.33; $p<0.001$ ), channeled laryngoscopes $(\mathrm{MD}=7.47 ; 95 \% \mathrm{Cl}: 2.59-12.34 ; \mathrm{p}=0.003)$, as well as fiberoptic laryngoscopes ( $M D=13.50$; 95\% Cl: 8.70-18.30; $p<0.001$ ).

The impact of PPE on endotracheal intubation success rate was recorded in eight studies $[12,17$, $18,21,22,25,27,30]$, and intubation without PPE was found to be superior to intubation with PPE in this regard (97.9\% vs. $90.0 \%$; $R R=0.94 ; 95 \% \mathrm{Cl}$ : 0.90-0.99; $p<0.001$ ) (Fig. 3).

In subgroup analysis, intubation without PPE was superior to intubation with PPE for all laryngoscope types: direct laryngoscopes (98.3\% vs. $89.3 \%$; $R R=0.93 ; 95 \% \mathrm{Cl}: 0.88-1.00 ; p=0.04)$, chan- neled laryngoscopes (96.1\% vs. 92.9\%; RR $=0.98$; 95\% Cl: 0.94-1.03; $p=0.49$ ), Macintosh blade laryngoscopes ( $100 \%$ vs. $73.3 ; \mathrm{RR}=0.74 ; 95 \% \mathrm{Cl}$ : $0.54-1.02 ; p=0.07)$, and fiberoptic laryngoscopes (100\% vs. 93.3\%; RR $=0.93 ; 95 \% \mathrm{Cl}: 0.83-1.05$; $\mathrm{p}=0.24)$.

\section{Direct laryngoscopy versus video laryngoscopy in personal protective equipment conditions}

Ten studies compared Macintosh laryngoscope with other laryngoscopes in PPE conditions [18, 19, 21, $23,27,28,30,31,34,35]$. Overall, intubation with direct laryngoscopes was shorter than that with video laryngoscopes (MD $=5.63 ; 95 \% \mathrm{Cl}$ : $-0.77-$ 12.03), although the difference was not statistically significant $(p=0.08)$ (Fig. 4). The subanalysis revealed that intubation with direct laryngoscopes was slightly faster than with Macintosh blade video laryngoscopes (MD $=-0.14 ; 95 \% \mathrm{Cl}:-5.61-5.33)$. For comparison of direct laryngoscopes with channelled laryngoscopes, faster intubation procedure was observed with direct laryngoscopy (MD $=6.41$; 95\% Cl: -2.41-15.24). However, the above differences were not statistically significant $(p=0.96$ and 
$\begin{array}{lll}\text { With PPE } & \text { Without PPE Risk Ratio Ratio }\end{array}$

Study or Subgroup Events Total Events Total Weight M-H, Random, $95 \% \mathrm{Cl}$ 1.1.1 Direct laryngoscopy

$\begin{array}{lrrrrr}\text { Castle 2009 } & 57 & 64 & 64 & 64 & 10.0 \% \\ \text { Castle 2011 (II) } & 61 & 66 & 66 & 66 & 11.5 \% \\ \text { Castle 2011 (McCoy) } & 54 & 66 & 65 & 66 & 7.7 \% \\ \text { Greenland 2007 } & 14 & 14 & 14 & 14 & 6.7 \% \\ \text { Koo 2018 } & 10 & 14 & 12 & 15 & 1.1 \% \\ \text { Plazik0wski 2018 } & 30 & 30 & 30 & 30 & 12.6 \% \\ \text { Shin 2013 } & 30 & 31 & 30 & 31 & 9.9 \% \\ \text { Taylor 2018 } & 12 & 15 & 15 & 15 & 2.3 \% \\ \text { Subtotal (95\% Cl) } & & 300 & & 301 & 61.7 \% \\ \text { Total events } & 268 & & 296 & & \end{array}$

Heterogeneity: Tau $^{2}=0.00 ; \mathrm{Chi}^{2}=18.30, \mathrm{df}=7(\mathrm{P}=0.01) ; \mathrm{I}^{2}=62 \%$

Test for overall effect: $Z=2.10(P=0.04)$

1.1.2 Channeled laryngoscopes

$\begin{array}{lrrrrr}\text { Castle 2011 (Il) } & 60 & 66 & 62 & 66 & 9.3 \% \\ \text { Plazikowski 2018 } & 27 & 30 & 29 & 30 & 6.5 \% \\ \text { Shin } 2013 & 31 & 31 & 31 & 31 & 12.8 \% \\ \text { Subtotal (95\% Cl) } & & 127 & & 127 & \mathbf{2 8 . 6 \%} \\ \text { Total events } & 118 & & 122 & & \end{array}$

$\begin{array}{lcc}\text { Total events } & 118 & 122 \\ \text { Heterogeneity: } \operatorname{Tau}^{2}=0.00 ; \mathrm{Chi}^{2}=1.41, \mathrm{df}=2(\mathrm{P}=0.49) ; \mathrm{I}^{2}=0 \%\end{array}$

Test for overall effect: $Z=0.69(P=0.49)$

1.1.3 Macintosh blade videolaryngoscopes

$\begin{array}{llllll}\text { Taylor } 2018 & 11 & 15 & 15 & 15 & 1.7 \% \\ \text { Subtotal }(95 \% \mathrm{Cl}) & & \mathbf{1 5} & & \mathbf{1 5} & \mathbf{1 . 7 \%} \\ \text { Total events } & 11 & & 15 & & \end{array}$

Total events 11

Test for overall effect: $Z=1.83(P=0.07)$

1.1.4 Fiberoptic laryngoscopes

$\begin{array}{lrlllll}\text { Plazikowski } 2018 & 28 & 30 & 30 & 30 & 8.0 \% & 0.93[0.83,1.05 \\ \text { Subtotal }(95 \% \mathrm{Cl}) & & 30 & & 30 & 8.0 \% & 0.93[0.83,1.05] \\ \text { Total events } & 28 & & 30 & & & \end{array}$

Heterogeneity: Not applicable

Test for overall effect: $Z=1.17(P=0.24)$

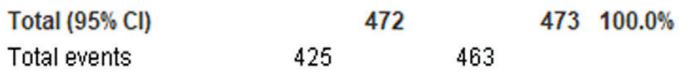

Heterogeneity: Tau $^{2}=0.00 ; \mathrm{Chi}^{2}=25.41, \mathrm{df}=12(P=0.01) ; \mathrm{I}^{2}=53 \%$

Test for overall effect: $Z=2.62(\mathrm{P}=0.009)$

Test for subaroup differences: $\mathrm{Chi}^{2}=4.33, \mathrm{df}=3(\mathrm{P}=0.23) . \mathrm{I}^{2}=30.7 \%$

$0.97[0.88,1.07]$

$0.93[0.81,1.07]$

$1.00[0.94,1.06]$

$0.98[0.94,1.03]$

$0.74[0.54,1.02]$

$0.74[0.54,1.02]$

$0.94[0.90,0.99]$ M-H, Random, $95 \% \mathrm{Cl}$

$0.92[0.86,1.00]$

$1.00[0.74,1.93]$

$0.89[0.59,1.35]$

$00[0.94,1.07]$

$0.81[0.61,1.06]$

$0.93[0.88,1.00]$

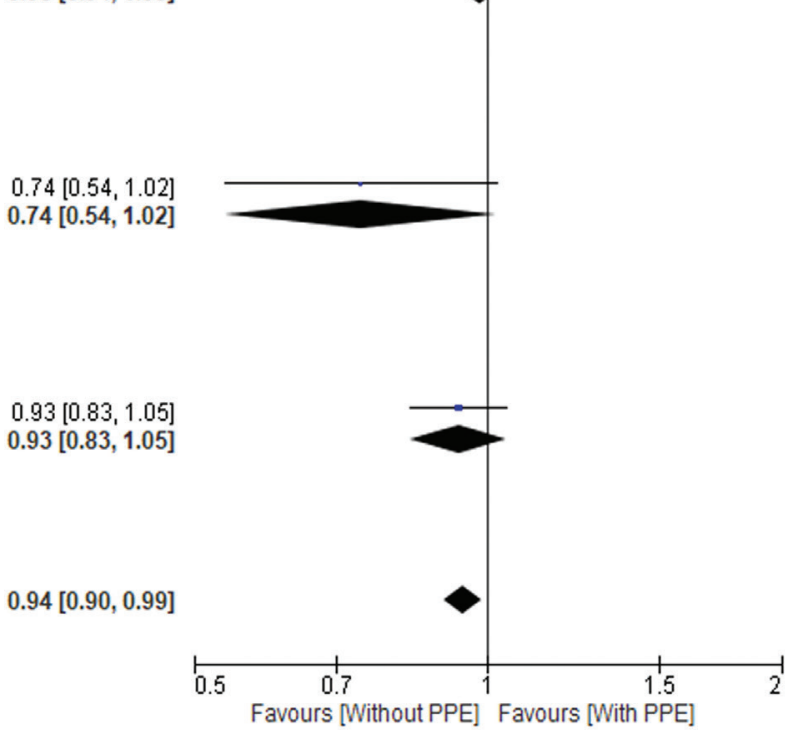

FIGURE 3. The impact of PPE on endotracheal intubation

$p=0.15$, respectively). In the case of fiberoptic laryngoscopes intubation, the duration of the procedure was statistically significantly longer than that of direct laryngoscopy (MD $=32.90 ; 95 \% \mathrm{Cl}$ : 28.5337.27; $p<0.001)$.

The intubation success rate for direct laryngoscopes versus other laryngoscopes in PPE conditions was reported in ten RCTs $[18,19,21,23,27,28$, $30,31,34,35]$. The effectiveness of intubation was comparable between direct laryngoscopes and video laryngoscopes ( $93.6 \%$ vs. $92.3 \% ; \mathrm{RR}=0.99 ; 95 \% \mathrm{Cl}$ : 0.96-1.02; $p=0.66$ ) (Fig. 5). Subgroup analysis showed that intubation with Macintosh blade video laryngoscopes was more effective than that with direct laryngoscopes (98.1\% vs. $96.4 \%$; RR $=1.00$; 95\% Cl: 0.97-1.03), although the difference was not statistically significant $(p=0.90)$. On the other hand, direct laryngoscope intubation was associated with higher efficiency as compared with channeled laryngoscopes (88.5\% vs. $91.2 \%$; RR $=0.99 ; 95 \% \mathrm{Cl}$ : $0.93-1.05 ; p=0.74)$ and fiberoptic laryngoscopes $(100 \%$ vs. $93.3 \%$; $R R=0.93 ; 95 \% \mathrm{Cl}: 0.83-1.05$; $\mathrm{p}=0.24)$.

Additional subanalysis with the division of operators into "Anesthesiology staff", "Emergency medicine staff", or "Mixed staff" revealed that in the first two groups, video laryngoscopy was associated with a longer procedure duration than direct laryngoscopy, while in the "Mixed staff" group, the opposite trend was observed (Tab. 2). Moreover, the analysis showed higher efficacy of direct laryngoscopy compared with video laryngoscopy (100\% vs. 96.8\%; $R R=0.97 ; 95 \% \mathrm{Cl}: 0.89-1.06 ; \mathrm{p}=0.50)$ (Tab. 3). For the "Emergency medicine staff", the efficacy 


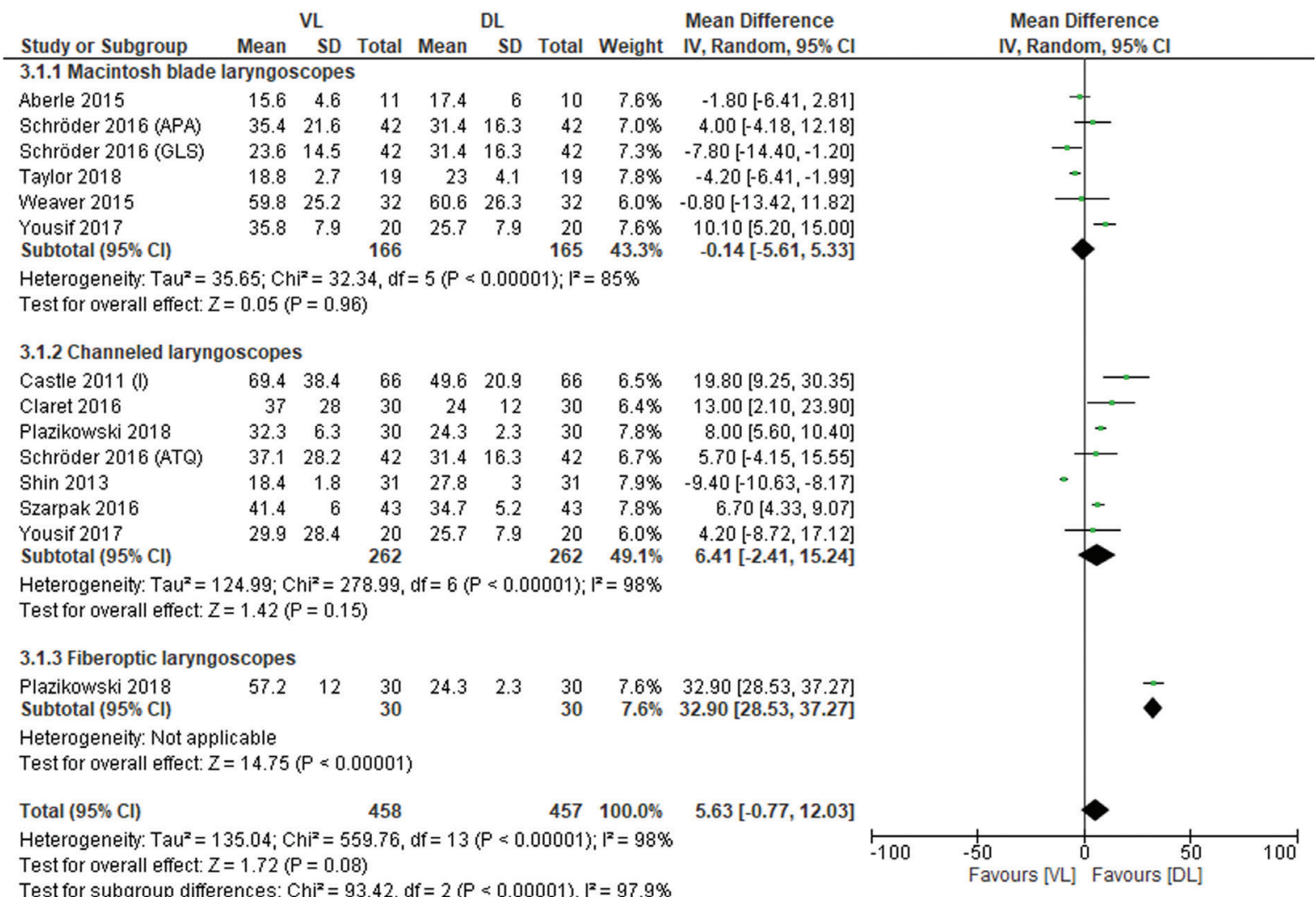

FIGURE 4. Intubation with direct laryngoscopes versus video laryngoscopes

with video laryngoscopy equalled $87.7 \%$ and was higher than that for direct laryngoscopy (87.3\%) $(\mathrm{RR}=1.02 ; 95 \% \mathrm{Cl}: 0.93-1.12)$. Among the "Mixed staff", the efficacy of direct laryngoscopy and video laryngoscopy intubation was $94.7 \%$ vs. $93.8 \%$ respectively.

\section{Risk of bias}

The risk of bias in the included studies is outlined in Supplementary digital content 1 and 2. All the 20 studies clearly described random sequence generation $[12,17-35]$. The risk of bias in the RCTs was assessed as either low or moderate across all domains, apart from the blinding of participants and personnel where blinding was clearly not possible.

\section{Limitations}

Our meta-analysis has some limitations. First, all the included studies were small and are at a high risk of bias as neither the operator nor the outcome assessor was blinded for obvious technical reasons. The second limitation is the influence of methodological heterogeneity from variations in the design of the original studies, such as involvement of diverse "patients" or different skill levels of operators; this heterogeneity should be perceived as an inherent limitation of meta-analysis. Third, not all studies reported intubation time and intubation success rate at the same time. Fourth, most of the studies included in the meta-analysis were simulation studies; however, owing to the risk of infection of medical personnel and the need to secure the airway as soon as possible, it would be impossible to conduct such studies in clinical conditions.

\section{DISCUSSION}

Endotracheal intubation is considered to be one of the basic procedures in the scope of emergency medicine and medical rescue, as well as during cardiopulmonary resuscitation. The comparison of endotracheal intubation with direct laryngoscopy and other intubation methods, including video laryngoscopy, has been widely studied and meta-analyzed. However, both the more common epidemics, including SARS and MERS, and the risk of infection with other dangerous pathogens, especially during 


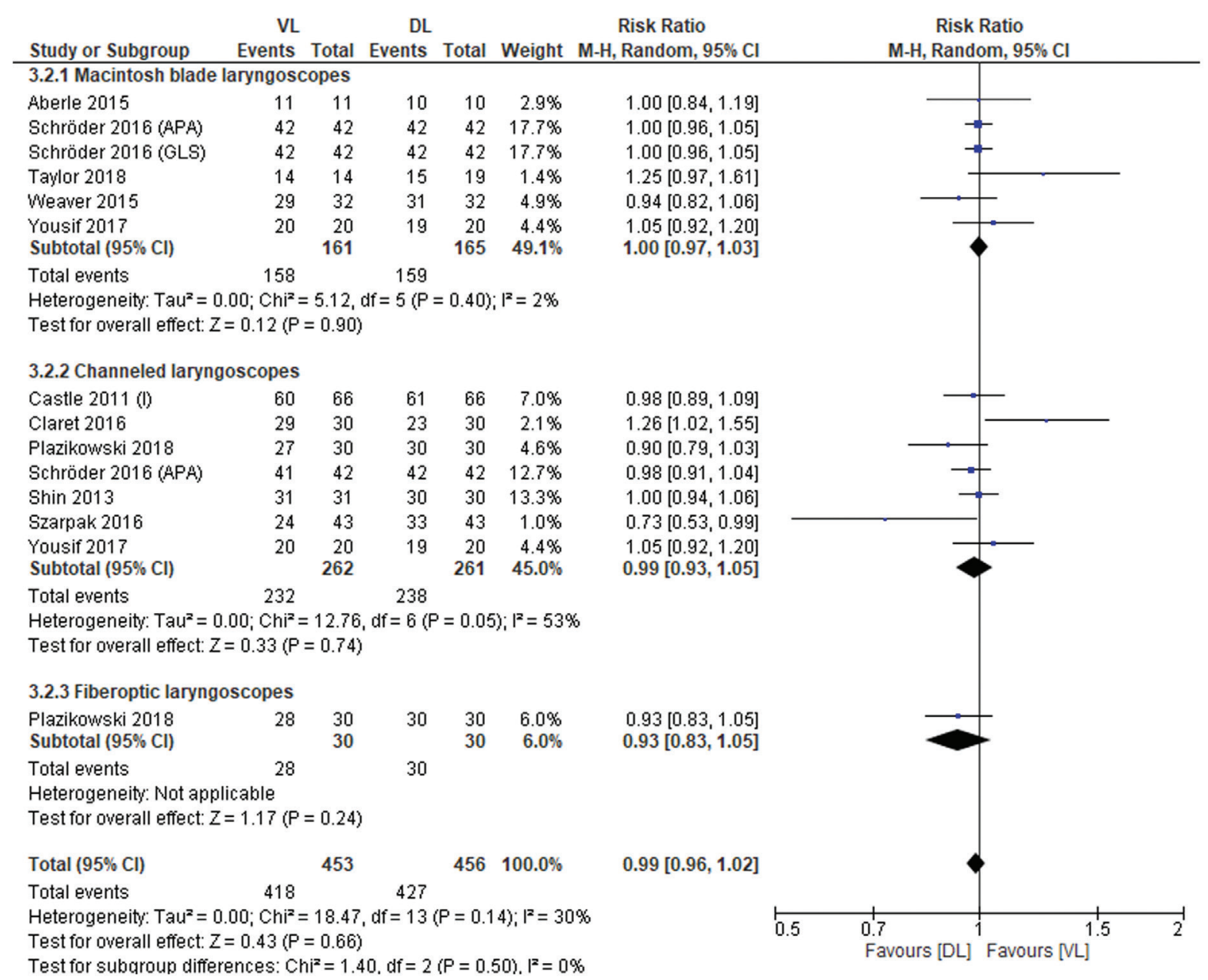

FIGURE 5. The effectiveness of intubation between direct laryngoscopes and video laryngoscopes

\begin{tabular}{|c|c|c|c|c|}
\hline & $\begin{array}{l}\text { Number } \\
\text { of trials }\end{array}$ & MD $(95 \% \mathrm{Cl})$ & $\begin{array}{c}P \\
\text { value }\end{array}$ & $\begin{array}{c}\mathrm{I}^{2} \\
\text { statistic, } \\
\%\end{array}$ \\
\hline $\begin{array}{l}\text { Anesthesiology } \\
\text { staff }\end{array}$ & 2 & $\begin{array}{c}10.27(0.44 \\
20.11)\end{array}$ & 0.04 & $97 \%$ \\
\hline $\begin{array}{l}\text { Emergency } \\
\text { staff }\end{array}$ & 6 & $\begin{array}{c}3.64(-1.99 \\
9.26)\end{array}$ & 0.20 & $90 \%$ \\
\hline Mixed staff & 2 & $\begin{array}{c}4.71(-23.89 \\
33.31)\end{array}$ & 0.75 & $97 \%$ \\
\hline
\end{tabular}

MD — mean differences; N/A — not applicable the current COVID-19 pandemic, suggest studies on the performance of medical procedures, also with reference to respiratory protective devices.

The number of available studies on respiratory protection under such conditions is limited and there are no meta-analyses of pooled data.

According to our knowledge, this was the first meta-analysis comparing Macintosh laryngoscope with video laryngoscopes in level C PPE conditions. We performed a priori subgroup analyses in order to investigate [1] the effect of PPE on intubation time and overall intubation success rate while

Table 3. Compared the video laryngoscopes with the Macintosh laryngoscope intubation success rate in
subgroup analysis
\begin{tabular}{|l|c|c|c|c|c|c|}
\hline & Number of trials & Effectiveness VL & Effectiveness DL & RR $(95 \%$ Cl) & P value & I2 statistic, \% \\
\hline Anesthesiology staff & 2 & $96.8 \%$ & $100 \%$ & $0.97(0.89,1.06)$ & 0.50 & $71 \%$ \\
\hline Emergency staff & 4 & $87.7 \%$ & $87.3 \%$ & $1.02(0.93,1.12)$ & 0.65 & $57 \%$ \\
\hline Mixed staff & 2 & $93.8 \%$ & $94.7 \%$ & $1.00(0.94,1.05)$ & 0.87 & $0 \%$ \\
\hline
\end{tabular}


using different types of laryngoscopes; [2] the effect of video laryngoscopy compared with direct laryngoscopy on intubation success rate and intubation time by type of video laryngoscopes under PPE conditions; [3] the influence of the type of operator on success rate and intubation time. Our study suggests that intubation with class $C$ protective suits has a statistically significant effect on prolonging the duration of the procedure and reducing its effectiveness. Moreover, the use of video laryngoscopes did not improve the overall success rate of endotracheal intubation when operators were wearing full PPE; on the contrary, video laryngoscopy intubation was associated with longer endotracheal intubation time and slightly lower efficacy compared with direct laryngoscopy. The analysis in subgroups showed only a slight advantage of Macintosh blade video laryngoscopes over direct laryngoscopy regarding the efficacy of intubation. Video laryngoscopes display the glottis on an external monitor by using a camera attached to the device blade without alignment of the oral-pharyngeal-tracheal axes.

Direct laryngoscopy also requires optimal head and neck positioning, proper insertion of the laryngoscope into the mouth, and glottis visibility, which demands a high level of operator experience [36]. Since video laryngoscopes - especially in conditions of difficult airways or difficult access to the patient - may offer better glottis visualization compared with direct laryngoscopes [37], they can facilitate endotracheal intubation, especially for less experienced staff.

The above relationships seem to be confirmed by numerous studies $[39,40]$. Additionally, as research indicates, the learning curve for video laryngoscopes is significantly shorter than for Macintosh or Miller laryngoscopes, which allows for effective endotracheal intubation by using video laryngoscopes after a short training $[41,42]$. The subanalysis of the study material showed that in the subgroup of "Emergency medicine staff", video laryngoscopy was associated with higher efficacy in comparison with direct laryngoscopy, but this difference was not statistically significant. Therefore, it may be inferred that for this professional group, including emergency physicians, paramedics, or emergency nurses, video laryngoscopy may be a good alternative to direct laryngoscopy for intubation under difficult conditions, which undoubtedly comprises intubation in full PPE.

A number of prospective and observational studies reveal that in emergency medicine conditions, the effectiveness of direct laryngoscopy intubation is insufficient $[36,43]$. As indicated in the study by Hoshijima et al. [44], another aspect that supports the use of video laryngoscopy, apart from the fact that it improves the visibility of the glottis, is that it significantly reduces the incidence of soft tissue bleeding compared with the Macintosh laryngoscope. Multiple attempts to intubate a patient may lead to desaturation and then intensify soft tissue bleeding and glottis edema, which in turn may result in a situation described by the Difficult Airway Society as "can't intubate, can't ventilate" [45]. Video laryngoscopes, owing to better visibility of the glottis compared with direct laryngoscopes, can reduce the risk of esophageal intubation in emergency and intensive care patients $[46,47]$.

\section{CONCLUSIONS}

Our meta-analysis suggests that PPE reduces the effectiveness of endotracheal intubation. The use of direct laryngoscopy for intubating patients with suspected/confirmed COVID-19 by an intubator wearing level C PPE is associated with overall intubation time reduction and an increase in intubation success rate compared with video laryngoscopes. However, the findings suggest that Macintosh blade video laryngoscopes during endotracheal intubation with PPE may be an alternative to direct laryngoscopes. Video laryngoscopy can be helpful for less experienced personnel.

Conflict of interest: The authors state no conflicts of interest.

\section{REFERENCES}

1. Szarpak L. Laryngoscopes for difficult airway scenarios: a comparison of the available devices. Expert Rev Med Devices. 2018; 15(9): 631-643, doi: 10.1080/17434440.2018.1511423, indexed in Pubmed: 30099914.

2. Madziala M, Okruznik $M$, Cobo $S A$, et al. Gold rules for pediatric endotracheal intubation. Am J Emerg Med. 2016; 34(8): 1711-1712, doi: 10.1016/j.ajem.2016.06.029, indexed in Pubmed: 27318745.

3. Natt BS, Malo J, Hypes CD, et al. Strategies to improve first attempt success at intubation in critically ill patients. Br J Anaesth. 2016; 117 Suppl 1: i60-i68, doi: 10.1093/bja/aew061, indexed in Pubmed: 27221259.

4. Russell TM, Hormis A. Rotherham NHS Foundation Trust. Should the Glidescope video laryngoscope be used first line for all oral intubations or only in those with a difficult airway? A review of current literature. J Perioper Pract. 2018; 28(12): 322-333, doi: 10.1177/1750458918788985, indexed in Pubmed: 30035689. 
5. Ruetzler K, Rivas E, Cohen B, et al. McGrath Video Laryngoscope Versus Macintosh Direct Laryngoscopy for Intubation of Morbidly Obese Patients: A Randomized Trial. Anesth Analg. 2020 [Epub ahead of print], doi: 10.1213/ANE.0000000000004747, indexed in Pubmed: 32175948.

6. Ruetzler K, Szarpak L, Filipiak K, et al. The COVID-19 pandemic - a view of the current state of the problem. Disaster and Emergency Medicine Journal. 2020, doi: 10.5603/demj.a2020.0015.

7. Szarpak L, Smereka J, Filipiak KJ, et al. Cloth masks versus medical masks for COVID-19 protection. Cardiol J. 2020 [Epub ahead of print], doi: 10.5603/CJ.a2020.0054, indexed in Pubmed: 32285928.

8. Li Li, Gong S, Yan J. Covid-19 in China: ten critical issues for intensive care medicine. Crit Care. 2020; 24(1): 124, doi: 10.1186/s13054-02002848-z, indexed in Pubmed: 32234064.

9. Smereka J, Szarpak L. COVID 19 a challenge for emergency medicine and every health care professional. Am J Emerg Med. 2020 [Epub ahead of print], doi: 10.1016/j.ajem.2020.03.038, indexed in Pubmed: 32241630 .

10. Chen $X$, Liu $Y$, Gong $Y$, et al. Chinese Society of Anesthesiology, Chinese Association of Anesthesiologists. Perioperative Management of Patients Infected with the Novel Coronavirus: Recommendation from the Joint Task Force of the Chinese Society of Anesthesiology and the Chinese Association of Anesthesiologists. Anesthesiology. 2020; 132(6): 1307-1316, doi: 10.1097/ALN.0000000000003301, indexed in Pubmed: 32195699.

11. Wax RS, Christian MD. Practical recommendations for critical care and anesthesiology teams caring for novel coronavirus (2019-nCoV) patients. Can J Anaesth. 2020; 67(5): 568-576, doi: 10.1007/s12630020-01591-x, indexed in Pubmed: 32052373.

12. Castle N, Owen R, Hann M, et al. Impact of chemical, biological, radiation, and nuclear personal protective equipment on the performance of low- and high-dexterity airway and vascular access skills. Resuscitation. 2009; 80(11): 1290-1295, doi: 10.1016/j.resuscitation.2009.08.001, indexed in Pubmed: 19709794.

13. Moher D, Shamseer L, Clarke M, et al. PRISMA-P Group. Preferred reporting items for systematic review and meta-analysis protocols (PRISMA-P) 2015 statement. Syst Rev. 2015; 4: 1, doi: 10.1186/20464053-4-1, indexed in Pubmed: 25554246.

14. Landis JR, Koch GG. The measurement of observer agreement for categorical data. Biometrics. 1977; 33(1): 159-174, indexed in Pubmed: 843571.

15. Higgins JPT, Altman DG, Gøtzsche PC, et al. Cochrane Bias Methods Group, Cochrane Statistical Methods Group. The Cochrane Collaboration's tool for assessing risk of bias in randomised trials. BMJ. 2011; 343: d5928, doi: 10.1136/bmj.d5928, indexed in Pubmed: 22008217.

16. Hozo SP, Djulbegovic B, Hozo I. Estimating the mean and variance from the median, range, and the size of a sample. BMC Med Res Methodol. 2005; 5: 13, doi: 10.1186/1471-2288-5-13, indexed in Pubmed: 15840177.

17. Koo A, Walsh R, Knutson T, et al. Comparison of Intubation Using Personal Protective Equipment and Standard Uniform in Simulated Cadaveric Models. Mil Med. 2018; 183(suppl_1): 216-218, doi: 10.1093/milmed/usx215, indexed in Pubmed: 29635606.
18. Scott Taylor R, Pitzer M, Goldman G, et al. Comparison of intubation devices in level C personal protective equipment: A cadaveric study. Am J Emerg Med. 2018; 36(6): 922-925, doi: 10.1016/j.ajem.2017.10.047, indexed in Pubmed: 29074070.

19. Aberle SJ, Sandefur BJ, Sunga KL, et al. Intubation Efficiency and Perceived Ease of Use of Video Laryngoscopy vs Direct Laryngoscopy While Wearing HazMat PPE: A Preliminary High-fidelity Mannequin Study. Prehosp Disaster Med. 2015; 30(3): 259-263, doi: 10.1017/ S1049023X15004707, indexed in Pubmed: 25959708.

20. Burns JB, Branson R, Barnes SL, et al. Emergency airway placement by EMS providers: comparison between the King LT supralaryngeal airway and endotracheal intubation. Prehosp Disaster Med. 2010; 25(1): 92-95, doi: 10.1017/s1049023×00007743, indexed in Pubmed: 20405470.

21. Castle N, Pillay $Y$, Spencer $N$. What is the optimal position of an intubator wearing CBRN-PPE when intubating on the floor: a manikin study. Resuscitation. 2011; 82(5): 588-592, doi: 10.1016/j.resuscitation.2011.01.005, indexed in Pubmed: 21330043.

22. Castle N, Pillay Y, Spencer N. Comparison of six different intubation aids for use while wearing CBRN-PPE: a manikin study. Resuscitation. 2011; 82(12): 1548-1552, doi: 10.1016/j.resuscitation.2011.06.020, indexed in Pubmed: 21763057.

23. Claret PG, Bobbia $X$, Asencio $R$, et al. Comparison of the Airtraq laryngoscope versus the conventional Macintosh laryngoscope while wearing CBRN-PPE. Eur J Emerg Med. 2016; 23(2): 119-123, doi: 10.1097/MEJ.0000000000000220, indexed in Pubmed: 25405463.

24. Garner A, Laurence $H$, Lee A. Practicality of performing medical procedures in chemical protective ensembles. Emerg Med Australas. 2004; 16(2): 108-113, doi: 10.1111/j.1742-6723.2004.00560.x, indexed in Pubmed: 15239724.

25. Greenland KB, Tsui D, Goodyear P, et al. Personal protection equipment for biological hazards: does it affect tracheal intubation performance? Resuscitation. 2007; 74(1): 119-126, doi: 10.1016/j.resuscitation.2006.11.011, indexed in Pubmed: 17353076.

26. Grillet G, Marjanovic N, Diverrez JM, et al. Intensive care medical procedures are more complicated, more stressful, and less comfortable with Ebola personal protective equipment: A simulation study. J Infect. 2015; 71(6): 703-706, doi: 10.1016/j.jinf.2015.09.003, indexed in Pubmed: 26391677.

27. Plazikowski E, Greif R, Marschall J, et al. Emergency Airway Management in a Simulation of Highly Contagious Isolated Patients: Both Isolation Strategy and Device Type Matter. Infect Control Hosp Epidemiol. 2018; 39(2): 145-151, doi: 10.1017/ice.2017.287, indexed in Pubmed: 29417920.

28. Schröder $H$, Zoremba $N$, Rossaint $R$, et al. Intubation performance using different laryngoscopes while wearing chemical protective equipment: a manikin study. BMJ Open. 2016; 6(3): e010250, doi: 10.1136/bmjopen-2015-010250, indexed in Pubmed: 27008688.

29. Schumacher J, Arlidge J, Garnham F, et al. A randomised crossover simulation study comparing the impact of chemical, biological, radiological or nuclear substance personal protection equipment on the performance of advanced life support interventions. Anaesthe- 
sia. 2017; 72(5): 592-597, doi: 10.1111/anae.13842, indexed in Pubmed: 28251618.

30. Shin DH, Choi PC, Na JiU, et al. Utility of the Pentax-AWS in performing tracheal intubation while wearing chemical, biological, radiation and nuclear personal protective equipment: a randomised crossover trial using a manikin. Emerg Med J. 2013; 30(7): 527-531, doi: 10.1136/ emermed-2012-201463, indexed in Pubmed: 23765764.

31. Szarpak L, Madziała M, Smereka J. Comparison of endotracheal intubation performed with 3 devices by paramedics wearing chemical, biological, radiological, and nuclear personal protective equipment. Am J Emerg Med. 2016; 34(9): 1902-1903, doi: 10.1016/j. ajem.2016.06.101, indexed in Pubmed: 27422219.

32. Udayasiri R, Knott J, MCD Taylor D, et al. Emergency department staff can effectively resuscitate in level $C$ personal protective equipment. Emerg Med Australas. 2007; 19(2): 113-121, doi: 10.1111/j.17426723.2007.00918.x, indexed in Pubmed: 17448096.

33. Wang $\mathrm{CC}$, Chaou $\mathrm{CH}$, Tseng $\mathrm{CY}$, et al. The effect of personal protective equipment on emergency airway management by emergency physicians: a mannequin study. Eur J Emerg Med. 2016; 23(2): 124-129, doi: 10.1097/MEJ.0000000000000157, indexed in Pubmed: 24858914.

34. Weaver KR, Barr GC, Long KR, et al. Comparison of airway intubation devices when using a biohazard suit: a feasibility study. Am J Emerg Med. 2015; 33(6): 810-814, doi: 10.1016/j.ajem.2015.02.051, indexed in Pubmed: 25817200.

35. Yousif S, Machan JT, Alaska Y, et al. Airway Management in Disaster Response: A Manikin Study Comparing Direct and Video Laryngoscopy for Endotracheal Intubation by Prehospital Providers in Level C Personal Protective Equipment. Prehosp Disaster Med. 2017; 32(4): 352-356, doi: 10.1017/S1049023X17000188, indexed in Pubmed: 28318455.

36. Suzuki K, Kusunoki S, Tanigawa K, et al. Comparison of three video laryngoscopes and direct laryngoscopy for emergency endotracheal intubation: a retrospective cohort study. BMJ Open. 2019; 9(3): e024927, doi: 10.1136/bmjopen-2018-024927, indexed in Pubmed: 30928937.

37. Hoshijima H, Mihara T, Maruyama K, et al. C-MAC videolaryngoscope versus Macintosh laryngoscope for tracheal intubation: A systematic review and meta-analysis with trial sequential analysis. J Clin Anesth. 2018; 49: 53-62, doi: 10.1016/j.jclinane.2018.06.007, indexed in Pubmed: 29894918.

38. Hoshijima H, Denawa Y, Tominaga A, et al. Videolaryngoscope versus Macintosh laryngoscope for tracheal intubation in adults with obesity:
A systematic review and meta-analysis. J Clin Anesth. 2018; 44: 69-75, doi: 10.1016/j.jclinane.2017.11.008, indexed in Pubmed: 29156438.

39. Breeman W, Van Vledder MG, Verhofstad MHJ, et al. First attempt success of video versus direct laryngoscopy for endotracheal intubation by ambulance nurses: a prospective observational study. Eur J Trauma Emerg Surg. 2020 [Epub ahead of print], doi: 10.1007/s00068-02001326-z, indexed in Pubmed: 32072225.

40. Min BC, Park JE, Lee GT, et al. C-MAC Video Laryngoscope versus Conventional Direct Laryngoscopy for Endotracheal Intubation During Cardiopulmonary Resuscitation. Medicina (Kaunas). 2019; 55(6), doi: 10.3390/medicina55060225, indexed in Pubmed: 31146497.

41. Sakles JC, Mosier J, Patanwala AE, et al. Learning curves for direct laryngoscopy and GlideScope ${ }^{\circledR}$ video laryngoscopy in an emergency medicine residency. West J Emerg Med. 2014; 15(7): 930-937, doi: 10.5811/westjem.2014.9.23691, indexed in Pubmed: 25493156.

42. Szarpak L, Smereka J, Ladny JR. Comparison of Macintosh and Intubrite laryngoscopes for intubation performed by novice physicians in a difficult airway scenario. Am J Emerg Med. 2017; 35(5): 796-797, doi: 10.1016/j.ajem.2017.01.005, indexed in Pubmed: 28139309.

43. Khan NU, Khan UR, Ejaz K, et al. Intubation in emergency department of a tertiary care hospital in a low-income country. J Pak Med Assoc. 2013; 63(3): 306-309, indexed in Pubmed: 23914625.

44. Hoshijima H, Mihara T, Maruyama K, et al. McGrath videolaryngoscope versus Macintosh laryngoscope for tracheal intubation: A systematic review and meta-analysis with trial sequential analysis. J Clin Anesth. 2018; 46: 25-32, doi: 10.1016/j.jclinane.2017.12.030, indexed in Pubmed: 29414609.

45. Collopy KT, Kivlehan SM, Snyder SR. Surgical cricothyrotomies in prehospital care. Surgical airway placement is indicated when you cannot intubate or ventilate. EMS World. 2015; 44(1): 42-49, indexed in Pubmed: 25803984.

46. Jiang J, Kang Na, Li Bo, et al. Comparison of adverse events between video and direct laryngoscopes for tracheal intubations in emergency department and ICU patients-a systematic review and meta-analysis. Scand J Trauma Resusc Emerg Med. 2020; 28(1): 10, doi: 10.1186/ s13049-020-0702-7, indexed in Pubmed: 32033568.

47. Michailidou M, O'Keeffe T, Mosier JM, et al. A comparison of video laryngoscopy to direct laryngoscopy for the emergency intubation of trauma patients. World J Surg. 2015; 39(3): 782-788, doi: 10.1007/ s00268-014-2845-z, indexed in Pubmed: 25348885. 